\title{
Accuracy and the Influence of Marrow Fat on Quantitative CT and Dual-Energy X-ray Absorptiometry Measurements of the Femoral Neck In Vitro
}

\author{
J. W. Kuiper ${ }^{1}$, C. van Kuijk ${ }^{1}$, J. L. Grashuis ${ }^{1}$, A. G. H. Ederveen ${ }^{2}$ and H. E. Schütte ${ }^{1}$ \\ ${ }^{1}$ Department of Experimental Radiology, Erasmus University Medical School and University Hospital 'Dijkzigt', Rotterdam; and \\ ${ }^{2}$ Department of Endocrinology, Organon Scientific Development Group, Organon International, Oss, The Netherlands
}

\begin{abstract}
Bone mineral measurements with quantitative computed tomography (QCT) and dual-energy X-ray absorptiometry (DXA) were compared with chemical analysis (ChA) to determine (1) the accuracy and (2) the influence of bone marrow fat. Total bone mass of 19 human femoral necks in vitro was determined with QCT and DXA before and after defatting. ChA consisted of defatting and decalcification of the femoral neck samples for determination of bone mineral mass $(\mathrm{BmM})$ and amount of fat. The mean $\mathrm{BmM}$ was $4.49 \mathrm{~g}$. Mean fat percentage was $37.2 \%(23.3 \%-48.5 \%)$. QCT, DXA and ChA before and after defatting were all highly correlated $(r>0.96, p<0.0001)$. Before defatting the QCT values were on average $0.35 \mathrm{~g}$ less than $\mathrm{BmM}$ and the DXA values were on average $0.65 \mathrm{~g}$ less than $\mathrm{BmM}$. After defatting, all bone mass values increased; QCT values were on average $0.30 \mathrm{~g}$ more than $\mathrm{BmM}$ and DXA values were $0.29 \mathrm{~g}$ less than BmM. It is concluded that bone mineral measurements of the femoral neck with QCT and DXA are highly correlated with the chemically determined bone mineral mass and that both techniques are influenced by the femoral fat content.
\end{abstract}

Keywords: Absorptiometry; Accuracy; Bone; Comparative study; Dual-energy X-ray; Femur; In vitro; Marrow fat, Quantitative CT

Correspondence and offprint requests to: Jan W. Kuiper, MD, Suite Ee 2338, Department of Experimental Radiology, Erasmus University Medical School, PO Box 1738, NL-3000 DR Rotterdam, The Netherlands. Fax: 31(0)10.436.8068.

\section{Introduction}

Single energy quantitative computed tomography (QCT) and dual energy X-ray absorptiometry (DXA) are commonly used non-invasive methods for measuring bone mineral content (BMC) and/or density (BMD). A reported disadvantage of QCT is the influence of the variable marrow fat content of bone on the accuracy of bone mineral measurements. Estimates of the accuracy-error of QCT measurements in various body sites range from $2 \%$ to $30 \%[1,2]$. Dual-energy QCT methods were suggested as a solution to the faterror problem and render higher accuracy, but at the cost of precision $[1,3]$. DXA and the preceding technique of dual photon absorptiometry (DPA) utilize a dual-energy method which separates soft tissue from bone but does not correct for marrow fat [4]. Various influences of marrow and soft tissue fat of DXA measurements on the spine have been reported [5-8]. Magnetic resonance studies show that the amount of marrow fat in the skeleton varies with age and location $[9,10]$ and is known to be influenced by state of health and medication [11].

The proximal femur, like the spine, is an area where many osteoporotic fractures occur. In particular the femoral neck has, unlike the spine, a complex geometry and a non-uniform distribution of bone mineral and marrow fat, which is likely to influence bone mineral measurements. Measurement of bone mineral in the proximal part of the femur with the use of DXA has become more or less standardized and generally applicable. Bone mineral measurements with QCT in the 
femur are still experimental. Various authors have presented QCT studies on the proximal part [12-14], the shaft [3] and on the distal part (e.g. condyles) $[15,16]$, but standardization of a QCT method for measuring bone mineral in the proximal femur has not been established.

The aim of this study was to evaluate the measurement of bone mass with QCT and DXA in the femoral neck and to compare the results with chemical analysis (ChA) in order to determine the accuracy of the bone mineral measurements. In addition, the influence of marrow fat on these measurements were investigated.

\section{Materials and Methods}

\section{Femoral Neck Specimen}

Nineteen femurs from embalmed human cadavers $(10$ males and 9 females, mean age $83.2 \pm 4.5$ years) were used. The proximal femurs were cleaned from all surrounding bones and soft tissues and mounted on a Perspex plate in a position comparable to the anatomical position of a recumbent patient. Long-distance radiographs of the mounted proximal femur were used to position the sawing lines for the femoral neck. Positioning of the sawing lines was done with the use of a template which had positioning lines perpendicular to the longitudinal axis of the femoral neck. These lines were drawn on the Perspex mounting plate. The femur was then frozen $\left(-80^{\circ} \mathrm{C}\right)$ to prevent loss of bone marrow during sawing, and a $20-\mathrm{mm}$ thick slice of the femoral neck was taken out with the use of a high-speed band saw. This $20-\mathrm{mm}$ thick slice was used for bone mass measurements using QCT, DXA and ChA.

\section{Chemical Analysis}

The individual constituent of bone (i.e. fat, bone mineral, collagen, etc.) were determined with ChA according to the following procedure. Directly after sawing the weight of bone samples was measured (WetM), and the samples were then degassed underwater at -0.95 bar for $24 \mathrm{~h}$ at room temperature. The bone samples were placed in the mounting device to prevent loss of bone marrow. After degassing, QCT and DXA scans (session 1; QCT 1 and DXA 1) were done. The samples were then dried under vacuum at a temperature of $45^{\circ} \mathrm{C}$ until constant weight was attained; this was considered dry mass (DryM1). Next the dried bones were defatted in $100 \%$ trichloroethylene for 2 weeks and subsequently dried and weighed again (DryM2). The defatted samples were again degassed under water and QCT and DXA measurements (session 2; QCT 2 and DXA 2) were done. After the second scan session the samples were dried and decalcified in decalcification fluid ( $37 \mathrm{~g} / 1$ sodium formiate and $170 \mathrm{ml} / 1$ formic acid in aqua dest) for 2 weeks with a fluid change after 1 week. Thereafter the remainder (OrgM) was dried again until constant weight; the bone mineral mass $(\mathrm{BmM})$ could then be calculated. The difference between DryM1 and DryM2 was considered to be the amount of fat (FatM). The amount of fat was also calculated as the percentage of WetM in a bone sample. This was considered to give the best representation of the physiological state of bone in vivo. OrgM was considered to be the organic constituents of the bone matrix. BmM was considered to be the amount of bone mineral in the bone sample.

\section{QCT Measurements}

A Perspex mounting device was developed for reproducible fixation of the femur specimen (Fig. 1, shaded part). This mounting device was placed in a circular water bath together with solid reference material. The femurs were degassed at room temperature, positioned in the mounting device and then scanned. CT scans were done using a Siemens Somatom Plus (Siemens, Erlangen, Germany) after calibration and quality checks of the CT system. Scanning parameters were: $80 \mathrm{kV}, 125$ $\mathrm{mA}, 1 \mathrm{~s}$ scan time. The water bath was positioned in the center of the gantry and 12 contiguous scans were done with a slice thickness of $2 \mathrm{~mm}$, ensuring complete scanning of the specimen. The first and the last scan were done through the Perspex plates which enclosed the femoral neck. To ensure a reproducible slice position in the specimen, the starting position was selected from a scout scan using a thin metal ring fixed on the mounting device as reference point.

From scans containing femoral neck images (generally 10), CT values of the object and reference material were obtained, using an ISG Allegro 3-D workstation with version 5.1 software. For seed-controlled contour detection of the femur a threshold of $78 \mathrm{HU}$ was selected. This threshold was used because it allowed an optimal distinction between the bone sample and the surrounding water bath. After a contour had been drawn the mean pixel value within this contour was determined. Solid reference material (Image Analysis

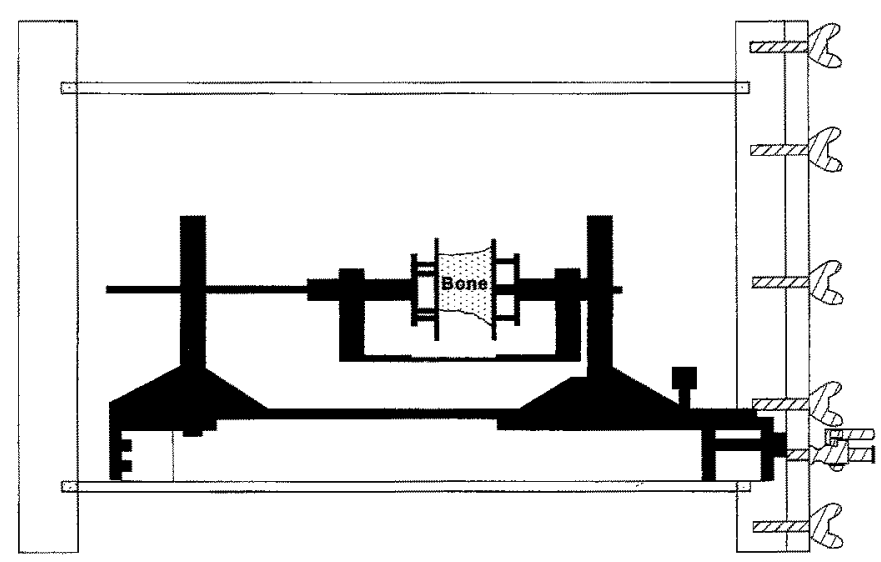

Fig. 1. Set-up of the mounting device and water bath for QCT measurements. The mounting device (shaded part) is shown with a sample of femur neck positioning in center (side view). 
Inc., Columbia, Ky) was used for calibration. Using the measured volume and BMD of all 2-mm scans the total bone mass of the femoral neck was calculated for sessions 1 and 2 .

\section{DXA Measurements}

The mounting device described above was also used for DXA measurements. It was placed in a square water bath with a water level of $15 \mathrm{~cm}$, stimulating soft tissue. Scans were done using a Lunar DPX-L scanner with version 1.3 software. After calibration and quality assurance tests, scans were done using the Spine scan option in Medium scan mode with a sample size of $1.2 \times$ $1.2 \mathrm{~mm}$ at $3000 \mu \mathrm{A}$ current. Spine scan option was used because there was no complete femur and it allowed a fixed start position and scans perpendicular to the longitudinal axis of the femoral neck. All scans were manually analyzed by the same person and the BMC (in grams) for each femoral neck specimen calculated.

\section{Statistical Analysis}

Statistical analysis was performed with the use of Statgraphics, Statistical Graphics System, version 6.0 software (STSC Inc., Rockville, MD).

\section{Results}

Results of ChA, QCT and DXA measurements are presented in Table 1; all weights are in grams. Correlations between QCT, DXA and BmM measurements before and after defatting were all high $(r>0.96)$, $p$ values $<0.0001$ ) .

\section{Accuracy}

The initial bone mineral measurements show that the mean QCT value (QCT 1) was $0.35 \mathrm{~g}$ less than mean $\mathrm{BmM}$ and the mean DXA value (DXA 1) was $0.65 \mathrm{~g}$ less than mean BmM. Compared with ChA-BmM, QCT 1 results show a consistent underestimation of $\mathrm{BmM}$, ranging from 0.31 to $0.37 \mathrm{~g}$ (Fig. 2). The slope of the regression line, at 0.984 , is close to optimal. An accuracy error for QCT can therefore be presented as a mean difference of $-0.35 \mathrm{~g}$ between QCT-BmM and ChA-BmM.

DXA 1 results also show an underestimation of ChA$\mathrm{BmM}$, ranging from 0.36 to $0.99 \mathrm{~g}$ (Fig. 3). The slope of the regression line, at 0.880 , is less optimal. DXA results tend to produce a larger underestimation of ChA-BmM at higher bone mass value. Thus, the DXA accuracy error is not a constant value and, therefore, can better be expressed as a relative value of $14.6 \%$ (percentage of ChA-BmM).

\section{Fat Influence}

Defatting of the femoral neck gave a mean fat percentage by weight of $37.2 \pm 7.9 \%$ with a range of $23.3 \%$ $48.5 \%$.

Compared with bone mass measurements before defatting, QCT and DXA showed an increase for both methods after defatting. The QCT 2 measurements increased $0.64 \mathrm{~g}$ and the values rose above the $\mathrm{ChA}$ -

Table 1. Results of ChA, QCT and DXA measurements (in grams)

\begin{tabular}{|c|c|c|c|c|c|c|c|c|c|c|}
\hline No. & WetM & DryM1 & DryM2 & FatM & OrgM & $\mathrm{BmM}$ & QCT 1 & $\mathrm{QCT}^{2}$ & DXA 1 & DXA 2 \\
\hline 1 & 23.78 & 16.94 & 9.15 & 7.80 & 4.06 & 5.09 & 4.85 & 5.41 & 4.23 & 4.56 \\
\hline 2 & 27.12 & 21.59 & 8.74 & 12.85 & 3.47 & 5.27 & 4.35 & 5.39 & 4.21 & 4.59 \\
\hline 3 & 23.56 & 19.23 & 8.07 & 11.16 & 3.48 & 4.59 & 4.46 & 5.01 & 3.85 & 4.37 \\
\hline 4 & 18.68 & 13.84 & 4.78 & 9.05 & 2.27 & 2.51 & 1.97 & 2.74 & 1.95 & 2.42 \\
\hline 5 & 23.55 & 18.28 & 7.44 & 10.84 & 3.31 & 4.14 & 3.67 & 4.62 & 3.25 & 4.03 \\
\hline 6 & 25.11 & 19.97 & 10.48 & 9.49 & 4.21 & 6.27 & 5.83 & 6.43 & 5.20 & 5.80 \\
\hline 7 & 21.26 & 17.01 & 11.66 & 5.35 & 4.34 & 7.32 & 6.92 & 7.22 & 6.66 & 6.67 \\
\hline 8 & 15.74 & 10.45 & 6.79 & 3.66 & 2.85 & 3.94 & 3.76 & 4.02 & 3.65 & 3.76 \\
\hline 9 & 21.63 & 15,60 & 8.98 & 6.62 & 3.94 & 5.04 & 4.38 & 4.87 & 4.18 & 4.61 \\
\hline 10 & 25.11 & 18.23 & 8.14 & 10.09 & 3.73 & 4.41 & 4.07 & 4.91 & 3.67 & 4.17 \\
\hline 11 & 11.25 & 9.01 & 3.75 & 5.27 & 1.65 & 2.10 & 1.76 & 2.16 & 1.74 & 1.95 \\
\hline 12 & 22.52 & 17.65 & 9.46 & 8.20 & 3.96 & 5.49 & 5.41 & 6.23 & 4.79 & 5.28 \\
\hline 13 & 27.09 & 19.83 & 10.23 & 9.60 & 4.59 & 5.64 & 5.29 & 6.16 & 5.00 & 5.28 \\
\hline 14 & 17.92 & 13.28 & 7.19 & 6.09 & 3.37 & 3.82 & 3.44 & 3.93 & 3.50 & 3.44 \\
\hline 15 & 16.13 & 10.29 & 6.22 & 4.07 & 2,68 & 3.54 & 3.31 & 3.62 & 3.17 & 3.27 \\
\hline 16 & 18.97 & 14.93 & 7.20 & 7.73 & 3.34 & 3.86 & 3.76 & 4.44 & 3.33 & 3.73 \\
\hline 17 & 19.45 & 15.03 & 7.33 & 7.70 & 3.23 & 4.10 & 3.94 & 4.60 & 3.45 & 3.88 \\
\hline 18 & 22.65 & 17.00 & 8.63 & 8.36 & 3.82 & 4.81 & 4.61 & 5.34 & 4.09 & 4.81 \\
\hline 19 & 13.98 & 10.47 & 5.98 & 4.50 & 2.67 & 3.31 & 2.88 & 3.78 & 3.04 & 3.19 \\
\hline Mean & 20.82 & 15.72 & 7.91 & 7.81 & 3.42 & 4.49 & 4.14 & 4.78 & 3.84 & 4.20 \\
\hline $\mathrm{SD}$ & 4.42 & 3.68 & 1.96 & 2.56 & 0.75 & 1.25 & 1.25 & 1.26 & 1.12 & 1.13 \\
\hline
\end{tabular}

For abbreviations see text. 
Fig. 2. Results of QCT versus ChA (BmM) measurements of femur neck. QCT 1, session 1 (scan results on complete bone sample); QCT 2, session 2 (scan results of defatted bone samples).

Fig. 3. Results of DXA versus ChA (BmM) measurements of femur neck. DXA 1, session 1 (scan results on com plete bone sample); DXA 2, session 2 (scan results of defatted bone samples).

Fig. 4. Differences between sessions 1 and 2 (normal and defatted bone respectively) as a percentage of the baseline measurement for QCT and DXA measurements versus fat percentage of the femoral neck determined by ChA.
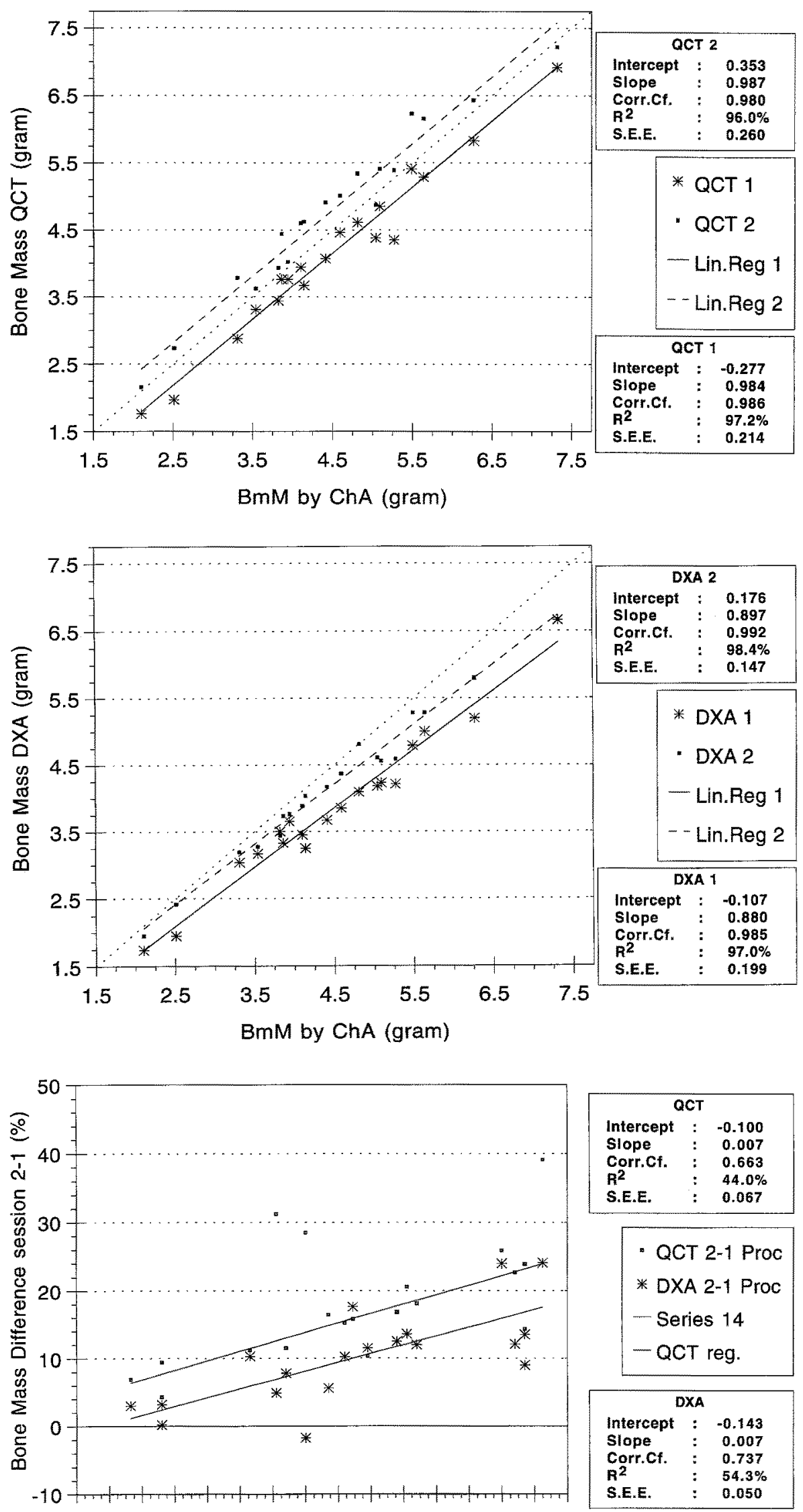

20222426283032343638404244464850

FAT Percentage (by weight) 
$\mathrm{BmM}$ values. The mean QCT 2 value was $0.30 \mathrm{~g}$ more than BmM (range 0.26-0.33 g, Fig. 2). The DXA measurements after defatting (DXA 2) increased by $0.36 \mathrm{~g}$ and still gave an underestimation of the $\mathrm{ChA}$ $\mathrm{BmM}$ values in the range 0.04-0.58 g (Fig. 3).

Figure 4 shows the percentage differences between measurements before and after defatting versus the fat percentage by weight. The regression lines show that for QCT the measurement error due to the variable amount of fat in the femoral neck ranges from $7.2 \%$ to $25.3 \%$. For DXA these values are between $1.2 \%$ and $17.6 \%$ respectively. In this study, the average increase in bone mass given as a percentage of the initially measured bone mass is, for both methods, about $0.7 \%$ per percentage increase in fat weight. This shows that when there is a higher fat percentage by weight in the femoral neck, the measurement errors of both QCT and DXA increase.

\section{Discussion}

In this study the QCT measurements were, as for DXA, for cortical and trabecular bone together. We consider that this is the only way to make an optimal comparison between the two radiological methods and ChA. An ashing method was not used on the bone samples. Our ChA has the advantage over ashing that the various compartments (e.g. water, fat, bone mineral and organic constituents) can be separated stepwise, thus allowing measurements at each stage.

There are two minor issues that were not considered in this study. We used material from embalmed human cadavers. Reports on the influence of the fixative on bone mineral measurements are lacking. We assume that this influence in our study is small and cannot account for differences between measurements before and after defatting.

Also, Whitehouse et al. [17] suggested that, when compared with in vivo measurements, temperature differences between objects and calibration material may give rise to an underestimation of accuracy errors (related to marrow fat) in QCT bone mineral measurements in vitro. This temperature factor will probably influence both QCT and DXA results in vitro. All our measurements were at room temperature and may therefore underestimate fat-related errors in vivo.

\section{Accuracy}

Our accuracy measurements showed that both methods underestimated the amount of bone mineral in the femoral neck. The trends show that QCT has a consistent underestimation while for DXA the measurement error increases at higher bone masses. The accuracy is influenced by a number of factors, one of which is the variable amount of marrow fat. Other, mainly technical factors are more or less the same for QCT and DXA.

For QCT, besides the variation by fat, the choice of calibration material may affect the initial underestimation and later the overestimation $[18,19]$. For DXA the exact calibration procedures are not clear but Mazess et al. [20] reported that the calibration material used is considered good when the fat content is about 1.5-2 times the mass of the ash content of the specimen. This does not explain the deviation at higher $\mathrm{BmM}$ values and the underestimation of the ChA-BmM values in our study, as the fat content was about 1.7 times the bone content. Ho et al. [21] reported comparable results for DXA of the spine, with a tendency to underestimate ash weight and an accuracy error of $8.9 \%$.

Other, possibly minor influences on the measurements include the partial volume effects and beam-hardening artifacts. Both QCT and DXA have the problem of partial volume effects. With QCT, using a $2 \mathrm{~mm}$ scan thickness, partial volume effects are inevitable in scans at the edges of the bone samples and these scans could not always be optimally analyzed. For DXA a scanline was $1.2 \mathrm{~mm}$ wide, and low scan profiles at the edges of the bone were excluded from measurement. Because of these partial volume effects there is small tendency for both methods to underestimate the chemically determined bone mass. These partial volume effects only influence the absolute accuracy, not the estimates of the fat error. The position of the calibration material for QCT measurements was inside the water tank and close to the (relatively small) object. Beam-hardening effects and field non-uniformity should, therefore, be minimal and/or have little effect on the QCT results. The existence of beam-hardening effects for DXA are reported by Goodsitt [7] and Blake et al. [22], but the magnitude of these errors in our study is unclear.

\section{Fat Influence}

In our bone samples we found a $25.2 \%$ variation in fat content. Both QCT and DXA are clearly influenced by the variable amount of fat and show an identical trend, with a $0.7 \%$ difference in measured bone mass for every $1 \%$ of fat weight. The only difference is the intercept of the regression lines. However, correlations and $R^{2}$ are relatively low and the values for both methods show considerable spread and overlap.

The QCT method used was a single-energy measurement. DXA is a dual-energy method, but should not be confused or compared with dual-energy QCT. Dualenergy QCT uses the two energies to estimate the fat content and the bone mineral content. DXA utilizes the dual energies for separation of the soft tissue from bone. It can roughly estimate the fat content of soft tissue, but cannot estimate the fat content of bone. According to Mazess et al. [20] a decrease of $0.05 \mathrm{~g} / \mathrm{cm}^{2}$ for each $1 \mathrm{~g} / \mathrm{cm}^{2}$ of fat can be expected with DXA measurements.

Our results show that, before and after defatting, both QCT and DXA are valid techniques for measuring bone mineral in the femoral neck. However, both 
techniques are influenced by the variable amount of marrow fat. The variation in amount of fat may not only be age specific but also exists between individuals. This will cause problems in longitudinal and cross-sectional measurements. Possible changes in amount of fat, as occurs in some therapies and diseases (e.g. corticosteroids, Gaucher diseases (Cushing disease) may also influence outcome of longitudinal bone mineral measurements.

The results of this study can provide basic information for use in interpreting future in vivo and clinical QCT and DXA measurements on the proximal femur. Further research is aimed at in vitro measurements with QCT and DXA on proximal femurs with the surrounding (pelvis) bone and soft tissue and at local variations in the amount of fat throughout the femoral neck.

Acknowledgements. We gratefully acknowledge the generous cooperation of the Department of Endocrinology, Organon International, Oss, The Netherlands, in performing the chemical analysis, and the Anatomical and Embryological Laboratory of the Academic Medical Centre, Amsterdam (Head: Prof. Dr R. Charles) for supplying embalmed femurs. Special thanks go to Mr C. A. C. Entius, Department of Anatomy, Erasmus University Rotterdam (Head: Prof. Dr J. Voogd), for his excellent work and assistance in preparing the femurs.

\section{References}

1. Cann CE. Quantitative CT for determination of bone mineral density: a review. Radiology 1988;166:509-22.

2. Revak CS. Mineral content of cortical bone measured by computed tomography. J Comput Assist Tomogr 1980;4:342-50.

3. Genant HK, Boyd DG. Quantitative bone mineral analysis using dual energy computed tomography. Invest Radiol 1977;12:54551.

4. Peppler WW, Mazess RB. Total body bone material and lean body mass by dual-photon absorptiometry. I. Theory and measurement procedure. Calcif Tissue Int 1981;33:353-9.

5. Hangartner TN, Johnston CC. Influence of fat on bone measurements with dual-energy absorptiometry. Bone Miner 1990;9:71-81.

6. Pye DW. Estimation of the magnitude of the error in bone mineral measurement due to fat: the effect of machine calibration. Clin Phys Physiol Meas 1991;12:87-91.
7. Goodsitt MM. Evaluation of a new set of calibration standards for the measurement of fat content via DPA and DXA. Med Phys 1991;19:35-44.

8. Tothill P, Avenell A. Errors in dual-energy X-ray absorptiometry of the lumbar spine owing to fat distribution and soft tissue thickness during weight change. Br $J$ Radiol 1994;67:71-5.

9. Dunnill MS, Anderson JA, Whithead R. Quantitative histological studies on age changes in bone. J Pathol Bacteriol 1967;94:275-91.

10. Ricci C, Cova M, Kang YS, Yang A, Rahmouni A, Scott WW, Zerhouni EA. Normal age-related pattern of cellular and fatty bone marrow distribution in the axial skeleton: MR imaging study. Radiology 1990;177:83-8.

11. Rosenthal DI, Hayes CW, Rosen B, Mayo-Smith W, Goodsitt MM. Fatty replacement of spinal bone marrow due to radiation: demonstration by dual energy quantitative CT and MR imaging. J Comput Assist Tomogr 1989;13:463-5.

12. Bhasin S, Sartoris DJ, Fellingham L, Zlatkin MB, André M, Resnick D. Three-dimensional quantitative CT of the proximal femur: relationship to vertebral trabecular bone density in postmenopausal women. Radiology 1988;167:145-9.

13. Esses SI, Lotz JC, Hayes WC. Biochemical properties of the proximal femur determined in vitro by single-energy quantitative computed tomography. J Bone Miner Res 1989;4:715-22.

14. Lotz J, Gerhart TN, Hayes WC. Mechanical properties of trabecular bone from the proximal femur: a quantitative $\mathrm{CT}$ study. J Comput Assist Tomogr 1990;14:107-14.

15. Braten M, Nordby A, Terjesen T, Rossvoll I. Bone loss after locked intramedullary nailing: computed tomography of the femur and tibia in 10 cases. Acta Orthop Scand 1992;63:310-4.

16. Alho A, Hoiseth A. Bone mass distribution in the lower leg: a quantitative computed tomographic study of 36 individuals. Acta Orthop Scand 1991;62:468-70.

17. Whitehouse RW, Economou G, Adams JE. Influence of temperature on QCT: implications of mineral densitometry. J Comp Assist Tomogr 1994;17;945-51.

18. Steenbeek JCM, Kuijk C van, Grashuis JL. Influence of calibration materials in single- and dual-energy quantitative $\mathrm{CT}$. Radiology 1992;183:849-55.

19. Faulkner KG, Glüer C-C, Grampp S, Genant HK. Crosscalibration of liquid and solid QCT calibration standards: corrections to the UCSF normative data. Osteoporosis Int $1993 ; 3: 36-42$.

20. Mazess RB, Tempe JA, Bisek JP, Hanson JA, Hans D. Calibration of dual energy X-ray absorptiometry for bone density. $\mathrm{J}$ Bone Miner Res 1991;6:799-806.

21. Ho CP, Kim RW, Schaffer MB, Sartoris DJ. Accuracy of dualenergy radiographic absorptiometry of the lumbar spine: cadaver study. Radiology 1990;176:171-3.

22. Blake GM, McKeeney DB, Chhaya SC, Ryan PJ, Fogelman I. Dual energy $X$-ray absorptiometry: the effects of beam hardening on bone density measurements. Med Phys 1992;9:459-65. 